\title{
A study on haematological and physiological effects of electromagnetic radiation from global system of communication
}

\author{
Mohd Rasheeduddin Imran ${ }^{1}$, Shweta K ${ }^{2, *}$, Aryan Singh ${ }^{3}$, Mohd Abrar Hassan ${ }^{4}$ \\ ${ }^{1,2}$ Assistant Professor, ${ }^{3}$ MBBS Student, ${ }^{4}$ Professor and HOD, Dept. of Physiology, Bhaskar Medical College, Yenkapally, \\ Telangana, India
}

*Corresponding Author:

Email: research.jan17@gmail.com

Received: $2^{\text {nd }}$ May, 2018

Accepted: $17^{\text {th }}$ May, 2018

\begin{abstract}
Aim: To study the effects of exposure to electromagnetic radiations from cell phone on Humans.

Materials and Methods: A prospective cross-sectional study was conducted in a secluded room of the Central Laboratory of the Medical College where use of electronic devises is prohibited. The study was conducted on 25 healthy male subjects and 25 health female subjects in the age range of 18-25 years of age. In post night fasting state, the study subjects were isolated in the secluded room for 3 hours to prevent any exposure to electromagnetic radiations. Blood sample is collected and divided into two aliquots, one is control sample (not exposed) and test sample (exposed to EMR with strength of $0.471 \mathrm{~W} / \mathrm{Kg}$ for $30 \mathrm{minutes}$ from a distance of $5 \mathrm{~cm}$ ). The samples were evaluated for blood cell count, blood indices and blood sugar levels. Nerve conduction study was done on median nerve prior to and after exposure to EMR for 5 minutes.

Results: Significant increase was observed in Mean Corpuscular Volume (MCV) (p 0.012) and Neutrophil count (p 0.026) in test sample compared to control sample. Significant decrease was observed in Mean Corpuscular Haemoglobin Concentration $(\mathrm{MCHC})(\mathrm{p} 0.049)$ and blood sugar levels $(\mathrm{p}<0.001)$ in test sample compared to control sample.

Nerve conduction study on median nerve has shown significant increase in conduction velocity (p 0.014).

Conclusion: The study has shown that even a limited exposure to EMR in recommended strengths can cause significant changes in haematological parameters and conduction velocity of nerve.
\end{abstract}

Keywords: Haematological, Electro magnetic radiations, Nerve conduction study.

\section{Introduction}

Radiofrequency Radiation (RFR) have long been used for different types of information exchange. Mobile phones, sometimes known as cellular or handset have hitherto lately formed an integral part of modern/ wireless telecommunication and are fast becoming a social lifestyle. ${ }^{1}$ Till the last decade, the cellular devices were affordable by only higher socioeconomic groups and the use was limited. The recent developments of technology has decreased the cost of the cellular communication device and made the device available easily to social class. This has increased the use of device and also the exposure to electromagnetic radiations from the cellular communication device has increased many folds.

In day to day life humans are exposed to electromagnetic radiation not only from Global system of communication but also from many other devices emitting such radiations. With increase in frequency and duration of use of cellular device, the high intensity radiations from very small distance can have adverse effect in humans.

In recent years, the public concern about the possible health hazards of electromagnetic waves in radio frequency field exposure for mobile phones has increased and initiated many studies on the possible adverse consequences on human health

Many studies had been done in animals to show adverse effects of the electromagnetic radiations. The results are positive in some studies while others have shown insignificant or negative correlation. The studies on the effects of these electromagnetic radiations in humans are limited. The possible interaction between the radio frequency electromagnetic radiations and its biological effects on human tissues particularly on the brain, cancer, and the human immune system has been investigated.

The radiation emanating from these communication devices have been found to be of a finite possibility in contributing adversely to the radiation exposure to man, thereby capable of affecting the health of the user. However, the mechanism of the interaction between the cellular phone systems and the effect of electromagnetic radiations still unclear.

There is possibility that the induced electric field due to exposure to EMF Radiation could cause cellular changes. Human body is a good conductor of electricity and therefore the electromagnetic waves can affect the electrical activity in excitable tissues of our body.

The present study is therefore designed to study the physiological and hematological effects of electromagnetic radiations from Global system of communication in humans.

\section{Materials and Methods}

The cross sectional study was done to investigate the various physiological and hematological parameters in human exposed to electromagnetic radiations from 
cell phone (Global system of mobile communication GSM). The study was conducted at Bhaskar General Hospital attached to Bhaskar Medical College.

This study was conducted on 25 healthy male subjects and 25 healthy female subjects in the age range of 18 to 25 years of age.

Materials: After obtaining informed written consent, detail history taken and physical examination was done in all subjects. The study evaluated the parameters in subjects in pre-exposure and post-exposure condition.

\section{Inclusion Criteria:}

1. Young healthy subjects

2. Age range of 18 to 25 years

3. No systemic disease

4. Non-smokers

5. Non-tobacco chewers

6. Non-alcoholics

Exclusion Criteria:

1. Subjects having systemic diseases

2. People with electronic and electrical implants

3. Occupation with EMW exposure

4. On medication or diet restriction

5. Alcoholics and tobacco smokers and chewers were excluded.

Methods: The subjects are instructed to stay fasting for 6 hours and isolated in the department laboratory to prevent any exposure to cell phone radiations. General physical examination was done and height, weight, Heart Rate Blood pressure were recorded.

Exposure Period: a standard exposure period of 30 minutes to the test sample on the day of investigation was taken for recording the post-exposure values of the parameters.

Exposure Strength: The specific absorption rate (SAR) level of the electromagnetic field inside the testing at $0.471 \mathrm{~W} / \mathrm{Kg}$ was simulated to determine the effect on the treated blood sample.

Precautions: An experienced technician was assisting the evaluation of Hematological parameters and an expert and experienced electrophysiologist was assisting in recording of the median nerve conduction velocity. The subjects were explained about the procedure and a written consent was obtained. The subjects and the technical staff were unaware of the aim and objective of the study.

\section{Measurement of Parameters}

Height and Weight: Height and weight was measured using a Detecto medical scale.

Blood Pressure: Systolic blood pressure (SBP) and diastolic blood pressure (DBP) were measured using a mercury sphygmomanometer. The blood pressure was recorded prior to the exposure and then after period of exposure to EMR

Pulse Rate: Single channel physiograph was utilized to measure heart rate. The pulse rate was recorded prior to the exposure and then after period of exposure to EMR.
The Electrophysiology Study: includes the evaluation of median nerve conduction study prior to and after exposure to EMR. This study was done in electrophysiology laboratory.

The nerve conduction study on the median verve is done using surface electrodes of the octopus 2 channel NCS machine and conduction velocity is recorded prior to the exposure and then after period of exposure to EMR.

Procedure: The subject sits with extended elbow, supinated foream and wrist in neutral position. The reference electrode is placed at distal interphalangeal joint of the thumb. The recording electrode is placed at the midpoint of the line drawn from the first metacarpophalangeal joint and the insertion of the tendon of the flexor carpus radialis. The site of stimulation is the wrist $80 \mathrm{~mm}$ proximal to the recording. The recording was taken prior to the exposure and then after period of exposure to EMR.

The Hematological Study: Includes the evaluation of blood cell counts, blood indices and blood sugar levels prior to and after exposure to EMR. This study was done using semi-automatic machine in pathology laboratory.

Collection of Blood Sample: Blood sample were collected under all aseptic precautions. All specimens were collected by cubital vein withdrawal procedure.

Each sample was then divided into two aliquots: control sample (sample not exposed) and experiment sample (sample exposed to mobile phone radiation).

A pre-exposure evaluation is done in the control and the experiment sample. The control samples were protected from exposure to the cell phone radiations and the other set was exposed to mobile phone radiations for 30 minutes from a distance of $5 \mathrm{~cm}$. At the end of exposure period all the hematological parameters were evaluated in both sets of samples.

Statistical Analysis: The effect of electromagnetic radiation exposure on different parameters between exposed and unexposed groups will be analyzed using Analysis of Variance (ANOVA). Data are reported as mean and standard deviation (SD) Students $\mathrm{T}$ test is done to know the significance using SPSS software analysis. A p Value less than 0.05 will be considered significant and less than 0.001 is considered highly significant.

\section{Results}

Comparison of pulse rate and blood pressure in pre-exposed condition and post-exposed condition to electromagnetic radiations from GSM. 
Table

\begin{tabular}{|l|c|c|c|c|c|c|}
\hline \multirow{2}{*}{ Parameters } & \multicolumn{2}{|c|}{ Pre-exposed } & \multicolumn{2}{c|}{ Post-exposed } & t-value & p-value \\
\cline { 2 - 7 } & MEAN & SD & MEAN & SD & & \\
\hline Pulse rate & 78.4 & 3.5023 & 78.8 & 4.2374 & -0.612 & 0.555 \\
\hline SBP & 112.4 & 7.1678 & 113.4 & 6.2574 & -1 & 0.343 \\
\hline DBP & 76.4 & 3.627 & 76.4 & 3.2386 & 0 & 1 \\
\hline
\end{tabular}

Comparison of median nerve conduction velocity in pre-exposed condition and post-exposed condition to electromagnetic radiations from GSM.

Table 2

\begin{tabular}{|l|c|c|c|c|c|c|}
\hline \multirow{2}{*}{ Parameters } & \multicolumn{2}{|c|}{ Pre-Exposed } & \multicolumn{2}{|c|}{ Post-Exposed } & t-value & p-value \\
\cline { 2 - 7 } & MEAN & SD & MEAN & SD & & \\
\hline $\begin{array}{l}\text { conduction } \\
\text { velocity }(\mathrm{m} / \mathrm{sec})\end{array}$ & 48.36 & 6.5187 & 48.487 & 6.5561 & -3.039 & 0.014 \\
\hline
\end{tabular}

Comparison of hematological parameters in pre-exposed condition and post-exposed condition to electromagnetic radiations from GSM.

Table 3

\begin{tabular}{|c|c|c|c|c|c|c|}
\hline \multirow[t]{2}{*}{ Parameters } & \multicolumn{2}{|c|}{ Pre-exposed } & \multicolumn{2}{|c|}{ Post-exposed } & \multirow[t]{2}{*}{ t-value } & \multirow[t]{2}{*}{ p- value } \\
\hline & MEAN & SD & MEAN & SD & & \\
\hline Total Leucocyte count & 6610 & 926.702 & 6630 & 990.0672 & 0.338 & 0.743 \\
\hline Red blood cell count & 5048000 & 390236.395 & 5040000 & 391265.752 & 0.54 & 0.602 \\
\hline Hemoglobin & 14 & 2.3399 & 13.96 & 2.331 & 1 & 0.343 \\
\hline Hematocrit & 45.89 & 9.5322 & 44.0 & 5.5507 & 0.943 & 0.37 \\
\hline $\begin{array}{l}\text { Mean Corpuscular } \\
\text { Volume }\end{array}$ & 86.93 & 8.1528 & 87.27 & 8.4223 & -3.127 & 0.012 \\
\hline $\begin{array}{l}\text { Mean Corpuscular } \\
\text { Hemoglobin }\end{array}$ & 27.72 & 3.9247 & 27.69 & 3.9354 & 0.394 & 0.703 \\
\hline $\begin{array}{l}\text { Mean Corpuscular } \\
\text { Hemoglobin } \\
\text { Concentration }\end{array}$ & 31.75 & 1.7964 & 31.58 & 1.7536 & 2.279 & 0.049 \\
\hline Platelet count & 300500 & 73513.793 & 300200 & 72592.928 & 0.089 & 0.931 \\
\hline Lymphocyte Percentage & 30.56 & 6.6658 & 30.13 & 6.5892 & 0.802 & 0.443 \\
\hline Neutrophil percentage & 59.83 & 5.425 & 60.80 & 6.0068 & -2.67 & 0.026 \\
\hline $\begin{array}{l}\text { Random Blood sugar } \\
\text { level }\end{array}$ & 84.30 & 7.8605 & 73.10 & 4.3063 & 7.074 & 0.0001 \\
\hline
\end{tabular}

\section{Observations}

Significant increase is seen in Mean Corpuscular volume (MCV) and Neutrophil percentage in test samples after exposure compared to control samples.

Significant decrease in Mean Corpuscular Hemoglobin Concentration (MCHC) and random Blood Sugar (RBS) levels in test samples after exposure compared to control samples.

There is also a significant increase in Median nerve conduction velocity

\section{Discussion}

In this study we have exposed the test sample to electromagnetic radiation from GSM cellular hand set
(Samsung duos) with specific absorption rate (SAR) of $0.471 \mathrm{~W} / \mathrm{Kg}$ for 30 minutes from a distance of $5 \mathrm{~cm}$. the control samples were isolated and prevented from exposure to any electromagnetic radiation from any devices from closed range (100 meters). The statistical analysis of the test sample results were also calculated and compared with controls. The objective of the study was to evaluate the physiological and hematological effects of electromagnetic radiation from global system of mobile Communication (GSM).

The study has shown that a brief exposure to electromagnetic radiation from a close range even though less than standard recommended dose can have significant changes in some physiological and 
hematological parameters. This study has shown that there is significant increase in Mean Corpuscular Volume (MCV) and Neutrophil percentage in test samples after exposure to the electromagnetic radiation. There was also significant decrease in Mean Corpuscular Hemoglobin Concentration (MCHC) and Random blood Sugar (RBS) levels.

The results of the hematological parameters of the exposed samples were compared and analyzed with that of the control samples using SPSS version 20. Significant difference was observed in some hematological variables within 30 minutes of exposure to electromagnetic radiation from GSM cellular handset.

There were no significant changes observed in blood pressure and pulse reat between the test and the control samples.

In one of the study the hematology results obtained due to long term exposure of Swiss Albino mice to RF EMF causes an increase in PCV, RBC and $\mathrm{HB}$ values with prolong exposure in all the exposed groups. However, MCHC and WBC show a decrease with prolong exposure in all the exposed groups. ${ }^{1}$ Some part of this study shows similar changes but with prolong exposure the variables may show different results.

The effects of electromagnetic radiation on biological subjects have been studied earlier and have reported changes in blood sugar levels. 5,10

Radiation from a cell phone $(850 \mathrm{MHz}, 2 \mathrm{~W}$ uniform electromagnetic field) either significantly decrease or increase the blood cell counts in both normal and high glucose blood samples and is a function of the power level and testing duration. ${ }^{8}$ This is in accordance with this study with decrease or increase the blood cell counts and changes in blood sugar levels.

The effects previously has been attributed to many factors, including i) false signal from oscillating ions that affect the electrochemical balance of the plasma membrane and cell function. ${ }^{11}$ ii) the cell membrane effect, ${ }^{6}$ iii) the dielectric and electrical properties of the blood. ${ }^{4,12}$ Discrepancies could also be due to the blood type, since blood samples used in each test come from different individuals, where the reaction mechanism is different, resulting in different response to the exposure.

Previous studies have concluded that electromagnetic fields are likely to affect the blood sugar properties, but it is difficult to point down the exact parameters that causes the effects. This is because of the different frequency, power levels and treatment durations involved.

While the limited sample size makes it difficult to say for certain that the results of this study gives a definitive differences between the tests/controls but some of the p-values were significant enough to suggest that the differences do exist, which suggests further investigation.

\section{Conclusion}

The study was done to evaluate the Hematological and Physiological effects of Electromagnetic radiation from global system of mobile communication (GSM). The study has shown that even a limited exposure to Electromagnetic radiations in recommended strengths can also cause significant changes when exposed from close distance. The study though done in vitro samples shows significant changes and indicates that safety measures are required to protect from in vivo changes that can be hazardous to general health condition of individuals. The result also signifies the changes that can occur in stored blood meant for transfusion and signifies the importance of protection of the stored blood in blood banks from Electromagnetic radiations. The electrophysiological changes occurring in the median nerve conduction velocity indicates that the excitable tissues, the nerves, the conducting system of heart, the brain tissue are in risk of altered functions if significant exposure occurs or with higher dosage of radiations.

\section{Summary}

Electromagnetic radiation has a negative impact in general health condition in humans and it can affect not only excitable tissues but also the body fluids. Many previous studies has shown that devices emitting Electromagnetic radiations causes significant changes in many systems in humans but certain other studies has shown insignificant effect.

This study was conducted in a group of 10 subjects who were regularly exposed to cell phone radiations. The subjects were in age range of 18-25 years and were free of any systemic disease.

The result indicates significant changes with increase in Mean Corpuscular Volume (MCV) and Neutrophil percentage in test samples after exposure to the electromagnetic radiations. There was also significant decrease in Mean Corpuscular Hemoglobin Concentration (MCHC) and Random Blood Sugar (RBS) levels.

These are acute changes occurring in the parameters and with increased exposure duration and frequency can cause significant changes in parameters that have shown insignificant changes.

Suggestions and Recommendations: This is a short term study with limited study group and limited exposure levels. But the significant changes in some of the Hematological and physiological parameters raises the concern about the long term effects of the exposure to electromagnetic radiations not only from Global system of communications but also from other devices emitting such radiations. The individuals staying near the base station with towers emitting larger doses of radiations are at higher risk.

The study therefore recommends limiting the use of such devices and also signifies the need of protective 
measures to prevent the individuals from harmful effects of the radiations. The recommendations suggested by A.S.M. iftekhar uddin jannatul ferdous in their study are important. (cell rad)

The study recommends further continuation of study with longer duration and study group for evaluation of many other systemic parameters such as hormonal changes, gonadal changes EEG changes etc.

Acknowledgements: We sincerely thanks ICMR for giving opportunity to conduct the research in Bhaskar Medical College and accepting and certifying the work. We sincerely thank the Management, Dean and Principal of Bhaskar Medical College for giving permission and support to conduct the study.

\section{References}

1. A.D Usaman, W.F Wan Ahmed, M.Z.A. Ab Kadir, M. Mokhtar and R Ariffin. Effect of radiofrequency Electromagnetic Field Explosure on Hematological Parameters of Mice. World Applied Sciences Journal. 2012;16(5):656-664.

2. Arash abdolmaleki, F Sanginabadi, et al. The effects of Electromagnetic waves exposure on blood parameters. International Journal of Hematology-Oncology and Stem Cell Research. 2012.6(2):13-16.

3. Ashraf A. Aly, Safaai Bin Deris, and Nazar Zaki. The effects on cells mobility due to exposure to EMF radiation. Advanced Computing: an International Journal (ACIJ), 2011;2(4):1-7.

4. Attia, A.A and M.A. Yehia, 2002 Histological ultrastructural and Immunohistochemical studies of the now frequency electromagnetic field effect on thymus, spleen and liver of albino Swiss mice. Pak J Biol Sci. 5(9):931-937.

5. Eraslan, G. et al. 2004 Effect of Short-Term Exposure to Pulsed Electromagnetic field on some Biochemical Parameters in Mice. Indian J. Biochemistry \& Biophysics, 2004;41:57-59.
6. Kumar. A, 2001 Electromagnetic Radiation and Biological Effects. IEEE Explore: Quebec, Canada.

7. Magda Havas. Radiation from wireless technology affects the blood, the heart and the autonomic nervous system. Rev Environ Health. 2013:28(2-3):75-84. DOI 10.1515/rveh-2013-0004

8. N. Boriraksantikul, K. D. Bhattacharyya, P.J.D. Whiteside C. O'Brien P. Kirawanich, J. A. Viator, and N. E. Islam. A case study of high blood glucose concentration effects of $850 \mathrm{mhz}$ electromagnetic fields using stem cell. Progress In Electromagnetic Research B, 2012;40, 55-77.

9. Olatunde Micheal Oni, Dauda Biodun Amuda, Celestime Etumonu Gilbert, lfe Fortunate Aseweje \& Felicia Adeola Akinola. A pilot study of the effects of GSM radiofrequency radiation on blood parameters. IJRRAS. 2011;9(3):464-466.

10. Prisco, M.G., et al., 2008 Effect of GSM- Modulated Radiofrequency Electromagnetic fields on Mouse Bone Marrow Cells. Radiat Res. 2008 Dec;170(6):803-10. doi: 10.1667/RR1213.1.

11. Rosen, A., M.A. Stuchly and A.V. Vorst, 2002. Application of RF/Microwaves in Medicine. IEEE Transaction on microwave theory and techniques, 2002;50(3):1044-1045.

12. Zsolt, F., et al., 2006 Effect of whole-body $1800 \mathrm{MHz}$ GSM-like microwave exposure on testicular steroidogenesis and histology in mice. Reprod Toxicol. $2006 \mathrm{Jul} ; 22(1): 111-7$.

How to cite this article: Imran MR, Shweta $\mathrm{K}$, Singh A, Hassan MA. A study on haematological and physiological effects of electromagnetic radiation from global system of communication. Ind $\mathrm{J}$ Clin Anat Physiol. 2018;5(3):397-401. 